\title{
Comparison of umbilical artery Doppler and non-stress test in assessment of fetal well-being in gestational diabetes mellitus: A prospective cohort study
}

Shirin Niromanesh ${ }^{1}$, Mahboobeh shirazi $^{1,2}$, Mitra Eftekhariyazdi $^{3}$, Forough Mortazavi $^{4}$

${ }^{1}$ Perinatologist, Professor, Maternal, Fetal and Neonatal Research Center, Women Hospital, Tehran University of Medical Sciences, Tehran, Iran

${ }^{2}$ Perinatologist, Associate Professor, Breast Feeding Research Center, Tehran University of Medical Sciences, Tehran, Iran

${ }^{3}$ Perinatologist, Assistant professor, Department of Obstetrics \& Gynecology, Faculty of Medicine, Sabzevar University of Medical Sciences, Sabzevar, Iran

${ }^{4}$ Ph.D. of Reproductive Health, Assistant professor, Department of Midwifery, School of Nursing \& Midwifery, Sabzevar University of Medical Sciences, Sabzevar, Iran

Type of article: Original

\begin{abstract}
Background: Gestational diabetes mellitus (GDM) is one of the most common medical complications of pregnancy and is related to poor perinatal outcomes. Reduction of neonatal complications of GDM is feasible by assessment of fetal well-being. Both fetal Doppler and NST are used for the screening of high-risk pregnancies.

Objective: We aimed to compare the non-stress test (NST) and umbilical artery (UA) Doppler assessments for evaluation of the adverse perinatal outcomes in GDM.

Methods: We conducted a prospective cohort study on 50 pregnant women with GDM in Jame Zanan Hospital, Tehran, Iran, from Oct 2014 to Sep 2015. A convenient sampling method was used for patient recruitment. Inclusion criteria were women with GDM, singleton pregnancies, and gestational age $>32$ weeks who had neither medical conditions nor fetal anomalies. Adverse perinatal outcomes were defined as Apgar scores at 1 -min and 5min $<7$, hypoglycemia (blood glucose $<45 \mathrm{mg} / \mathrm{dl}$ ), neonatal acidosis $(\mathrm{PH}<7.2)$, hypocalcemia $(\mathrm{Ca}<8 \mathrm{mg} / \mathrm{dl}$ ), admission to the NICU for more than 24 hours, and perinatal death. Statistical analyses were performed with SPSS version 16 using Chi-square, Fisher's exact test, and independent-samples t-test. The significance level was considered at 0.05 .

Results: Totally, $22 \%$ and $12 \%$ of women had an abnormal UA Doppler and a non-reactive NST respectively. Poor outcomes were detected in 13 women. The most frequent poor outcomes were hypoglycemia $(\mathrm{n}=9)$, Apgar 1-min $<7(n=8)$, neonate admitted in NICU $(n=6)$, and respiratory distress syndrome $(n=6)$. Poor outcome was more prevalent in women with non-reactive NST $(\mathrm{p}<0.001)$, abnormal UA Doppler $(\mathrm{p}=0.033)$, and those with infant birth weight $>4000$ gram $(\mathrm{p}=0.033)$. Sensitivity and specificity of the NST in predicting different poor outcomes were $76.9 \%$ and $97.3 \%$ respectively. Sensitivity and specificity of UA Doppler in predicting different poor outcomes were $30.8 \%$ and $94.6 \%$ respectively.

Conclusion: NST is a better predictor of adverse perinatal outcomes in GDM patients.

Keywords: Diabetes, Gestational, Umbilical arteries, Ultrasonography, Prenatal diagnosis
\end{abstract}

\section{Introduction}

Gestational diabetes mellitus (GDM) is one of the most common antenatal pregnancy complications. GDM is actually a condition of carbohydrate intolerance that starts or is first recognized during pregnancy $(1,2)$. Approximately $7 \%$ of all pregnancies are complicated by GDM. The prevalence may range from 1 to $14 \%$ of all

\section{Corresponding author:}

Assistant Professor Dr. Mitra Eftekhariyazdi, Department of Obstetrics \& Gynecology, Faculty of Medicine, Sabzevar University of Medical Sciences, Sabzevar, Iran.

Tel: +98.9155710054, Fax: +98.5144664222, Email: m_eftekhari1144@yahoo.com

Received: October 26, 2016, Accepted: September 20, 2017, Published: December 2017

iThenticate screening: August 29, 2017, English editing: December 02, 2017, Quality control: December 15, 2017

This article has been reviewed / commented by three experts

(C) 2017 The Authors. This is an open access article under the terms of the Creative Commons Attribution-NonCommercialNoDerivs License, which permits use and distribution in any medium, provided the original work is properly cited, the use is non-commercial and no modifications or adaptations are made. 
pregnancies (3). About $90 \%$ of patients with diabetes diagnosed during pregnancy are cases of GDM (1). GDM has both maternal and neonatal complications. Uncontrolled GDM has profound effects on fetal growth and neonatal health; however, maternal and neonatal outcomes in pregnancies with controlled GDM was higher than in those with high-risk pregnancies without GDM (4). Neonatal complications consist of hypoglycemia, hypocalcemia, hyper bilirubinemia, polycythemia, operative delivery, shoulder dystocia, macrosomia, birth injury, asphyxia, and respiratory distress syndrome (RDS) (1). Diagnosing, monitoring and controlling of GDM as well as assessment of fetal well-being may reduce the impact of diabetes on the fetus and decrease neonatal complications $(1,5)$. The nonstress test (NST) has been used for assessing fetal well-being in high-risk pregnancies (6). More recently, Doppler has been applied to the screening of poor outcomes in high-risk pregnancies (7-9). The NST is a noninvasive prenatal test and used for monitoring the fetal heart rate in response to the fetal movements. An NST does not place any stress on the fetus during the test and can be done by a midwife. Gynecologists recommend the NST when the fetus is at increased risk of death in high-risk pregnancies during the third trimester of pregnancy. The NST provides a continuous record of the fetal heart rate obtained by an ultrasound transducer placed on the mother's abdomen (10). A non-reactive test is sometimes associated with poor fetal or neonatal outcomes, while a reactive test is usually associated with a healthy fetus (11). Previous study showed that NST, especially by remote electronic fetal monitoring network, could improve perinatal outcome in GDM patients (12). In addition, NST was recommended for fetal surveillance as a choice method in diabetic patients in low resource settings (13). A UA Doppler ultrasound is also a noninvasive test that is used to estimate the blood flow through umbilical artery and discloses placental vascular resistance in the third trimester of pregnancy. A UA Doppler ultrasound does not induce any stress on the fetus during the test but a perinatologist must perform the test. Abnormal umbilical artery Doppler is a marker of utero-placental insufficiency and consequent intrauterine growth restriction (IUGR). Abnormal waveforms include elevated systolic/diastolic (S/D) umbilical arterial ratio as well as absent and reversed end-diastolic velocimetry. The use of routine UA Doppler ultrasound in low-risk women has not been supported (14). The study by Maulik et al. confirmed the benefit of UA Doppler assessment for prediction of adverse perinatal outcome in high-risk pregnancy. They found that an increased UA Doppler index and absent and reversed end-diastolic velocimetry were related to fetal hypoxia, low Apgar scores, decreased cord $\mathrm{PH}$, meconium staining and admission to neonatal intensive care unit (NICU) (15). Some studies compared these two popular methods and found opposing results. Williams et al (2003) compared the NST with UA Doppler assessment of the fetus and concluded that the NST resulted in a decreased cesarean delivery rate for fetal distress in high-risk pregnant women (16), while Bracero et al (1996) showed that UA Doppler assessment was superior to NST in identifying adverse outcomes in diabetic patients (17). For these reasons and because of contradictory data and the prevalence of GDM in Iran which is reported to be between 1.3 and $8.9 \%$ (18), this study aimed to compare NST and UA Doppler assessments for evaluation of the adverse perinatal outcomes in women with GDM.

\section{Material and Methods}

\subsection{Study design and subjects}

The present research was a prospective cohort study that was carried out from October 2014 through September 2015 in the Department of Gynecology and Obstetrics of Jame Zanan Hospital which is affiliated with Tehran University of Medical Sciences (TUMS), Tehran, Iran. The study population consisted of 50 women with GDM between 32 and 40 weeks gestation. The gestational age (GA) was determined either by the LMP or by the measurement of fetal crown-rump length at 11-13 weeks or by fetal head circumference at 19-24 weeks. A convenience sampling method was used to select patients. Inclusion criteria were women with GDM and singleton pregnancies with intact membranes. Women with other medical conditions that affect perinatal outcomes or those with fetal anomalies detected in the first half of the pregnancy were excluded from the study. The diagnosis of GDM was made based on American College of Obstetricians and Gynecologists Guidelines (19). Women who had been screened in prenatal visits with a Glucose Challenge Test (GCT) $\geq 140 \mathrm{mg}$ were referred to the hospital clinic where Glucose Tolerance Tests (GTT) were performed at weeks $24-28$. The diagnosis of GDM was labeled when at least two measurements exceeded the cut-off. The cut-offs were a GTT $\geq 95,180,155$, and 140 in fasting, one, two, and three hours after oral intake of $100 \mathrm{mg}$ glucose respectively.

\subsection{Data collection}

A questionnaire including socio-demographic history, obstetric history, and laboratory tests was designed. Data on age, gravidity, parity, and GA was gathered. At the first visit, patients' diabetic histories was taken and physical examinations were performed. Laboratory tests consisting of fasting blood sugar, 2-hour postprandial blood sugar, complete blood count, kidney and liver function tests, urine analysis and ultrasonography studies were requested every month. Adverse perinatal outcomes were defined as Apgar scores at 1-min and 5-min <7, hypoglycemia 
(blood glucose $<45 \mathrm{mg} / \mathrm{dl}$ ), neonatal acidosis ( $\mathrm{PH}<7.2$ ), hypocalcemia $(\mathrm{Ca}<8 \mathrm{mg} / \mathrm{dl}$ ), admission to the NICU for more than 24 hours, and perinatal death. Apgar scores were determined at 1 minute and 5 minutes. The 1 -minute Apgar score was measured to determine the need for resuscitation and the 5-minute Apgar for the evaluation of neonatal morbidity and mortality. The UA Doppler assessment was conducted weekly. The last assessment just before termination of pregnancy was considered for comparisons. UA Systolic/Diastolic $\geq 3$ was considered abnormal. Cesarean was performed when an absent or reversed umbilical artery end-diastolic flow was seen. Physical examination of each neonate was carried out and the condition evaluated. UA PH was ascertained immediately after delivery, 1 and 5 minute Apgar scores were recorded, and finally fetal acidosis, blood sugar and calcium were checked. The NST was performed weekly. Result of the test can be reactive or nonreactive. When there are two or more fetal heart rate accelerations that peak at least 15 beats per minute above the baseline and last at least 15 seconds from baseline following fetal movements, the NST is considered reactive (20). In cases of no fetal movement, the NST can be repeated. Women with a non-reactive test were examined in further tests. The last NST was considered for comparisons.

\subsection{Equipment}

The ultrasound equipment used was the Acuson Antares 500, Siemens AG 2011, 3.5-5 MHZ probe with pulsed wave and color Doppler modes with high resolution. All measurements were performed and were recorded in three consecutive waveforms by the main investigator when the fetus was not moving or breathing. The mean of three measurements were recorded. The NST equipment was a fetal monitor manufactured by Bistos Co., Ltd, model name: BT-330. For assessment of the fetal well-being, a fetal monitor records fetal heart rate for 20 minutes.

\subsection{Statistical analysis}

SPSS version 18 was used for data analysis. We assessed the normality of data with Shapiro-wilk test. Gestational age (GA) and birth weight were normally distributed. Results were expressed as Mean $\pm \mathrm{SD}$ and percentages. Independent-samples t-test was used for comparing gestational age and birth weight between poor and good outcome groups. The relation between outcome variables and UA or NST tests were assessed by Chi-square or Fisher's exact test. The significance level was considered 0.05 . The validity of both tests was examined by determining sensitivity and specificity of tests in predicting adverse outcomes. The sample size was determined based on previous studies and the time limit for completing a fellowship.

\subsection{Ethics}

The ethics committee of the medical school of Tehran University of Medical Sciences approved the study protocol. In addition, all of the enrolled women were verbally informed about the project and they all signed a written consent form. Patients received the best medical care available in Iran. Since the study was an observational one, patients received no extra test unrelated to their treatment process, and were not required to pay any extra charges.

\section{Results}

Evaluations were made of 50 pregnant patients with GDM. The mean age, gestational age, and birth weight were $31.5 \pm 6$ years, $38.6 \pm 1.2$ weeks, and $3555 \pm 343$ grams. The most frequent poor outcomes were hypoglycemia $(\mathrm{n}=9)$, Apgar 1-min $<7(n=8)$, neonate admitted in NICU $(n=6)$, and respiratory distress syndrome $(n=6)$. Only one patient was under insulin treatment. Poor outcome was more prevalent in women with non-reactive NST $(\mathrm{p}<0.001)$, abnormal UA Doppler ( $\mathrm{p}=0.033)$, and those with infant birth weight $>4000$ gram $(\mathrm{p}=0.033)$ (Table 1).

Table 1. Sample Characteristics in Good and Poor Neonatal Outcomes

\begin{tabular}{|l|l|l|l|l|}
\hline Variables & \multirow{2}{*}{ Mean \pm SD / n (\%) } & \multicolumn{2}{l|}{ Neonatal Outcome } & \multirow{2}{*}{-value } \\
\cline { 3 - 4 } & & Good $(\mathrm{n}=37)$ & Poor $(\mathrm{n}=13)$ & \\
\hline Age (year) & $31.5 \pm 6.0$ & $31.7 \pm 6.3$ & $31.0 \pm 5.6$ & 0.712 \\
\hline Primigravidity & $37(74)$ & $28(56)$ & $9(18)$ & 0.453 \\
\hline Nulliparity & $27(54)$ & $23(46)$ & $4(8)$ & 0.051 \\
\hline Cesarean & $19(38)$ & $13(26)$ & $6(12)$ & 0.351 \\
\hline Fast Blood Sugar (FBS) $\geq 95 \mathrm{mg}$ & $12(24)$ & $7(14)$ & $5(10)$ & 0.149 \\
\hline Blood Sugar $(\mathrm{BS}) \geq 120 \mathrm{mg}$ & $24(48)$ & $15(30)$ & $9(18)$ & 0.072 \\
\hline Gestational age (week) & $38.6 \pm 1.2$ & $38.8 \pm 0.7$ & $38.2 \pm 2.1$ & 0.300 \\
\hline Birth weight $>4000$ gram & $6(12)$ & $2(4)$ & $4(8)$ & 0.033 \\
\hline Non-reactive NST $\dagger$ & $11(20)$ & $1(2)$ & $10(22)$ & $<0.001$ \\
\hline Abnormal UA Doppler $\ddagger$ & $6(12)$ & $2(4)$ & $4(8)$ & 0.033 \\
\hline
\end{tabular}

$\dagger$ Non-Stress Test, $\ddagger$ Umbilical Artery Doppler 
http://www.ephysician.ir

There was significant difference between 1-minute and 5-minute Apgar score mean in two groups (good vs. poor outcome) $(\mathrm{p}=0.002, \mathrm{p}=0.005)$. There was significant difference between 1 -minute and 5-minute Apgar score mean in fetuses with reactive vs. non-reactive NST $(p=0.004,0.01)$. Sensitivity and specificity of UA Doppler and NST in predicting neonatal outcomes is compared in Table 2. Table 3 shows the distribution of NST and UA Doppler results according to neonatal outcomes. Poor outcome was observed despite normal NST and normal Doppler in two cases. Non-harmonious result is observed in the poor outcome group where seven patients with a non-reactive NST had a normal UA; however, a Mantel-Haenszel test indicates that the results of the UA and NST tests were in harmony in good and poor outcome groups $(\mathrm{p}=0.859)$ which may be due to low sample size.

Table 2. Comparison of UA and NST Doppler Validity in predicting Neonatal Outcomes

\begin{tabular}{|c|c|c|c|c|c|c|c|}
\hline Group & $\begin{array}{l}\text { Reactive NST } \\
(\mathrm{n}=39) ; \mathrm{n}\end{array}$ & $\begin{array}{l}\text { Non-reactive } \\
\text { NST }(\mathrm{n}=11) ; \mathrm{n}\end{array}$ & p-value & $\begin{array}{l}\text { Sensitivity; } \\
\%\end{array}$ & $\begin{array}{l}\text { Specificity; } \\
\%\end{array}$ & $\begin{array}{l}\text { Positive } \\
\text { Predictive Value; } \\
\%\end{array}$ & $\begin{array}{l}\text { Negative } \\
\text { Predictive Value; } \\
\%\end{array}$ \\
\hline Acidemia & 0 & 0 & - & - & - & & \\
\hline RDS† & 1 & 5 & $0.001 * * *$ & 83.3 & 86.4 & 45.5 & 97.4 \\
\hline Neonatal death & 0 & 1 & 0.220 & 100 & 79.6 & 9.1 & 100 \\
\hline Hypocalcaemia & 0 & 2 & $0.045^{*}$ & 100 & 81.3 & 18.2 & 100 \\
\hline Hypoglycemia & 3 & 6 & $0.002 *$ & 66.6 & 87.8 & 54.5 & 92.3 \\
\hline NICU admission & 1 & 5 & $0.001 * * *$ & 83.3 & 86.4 & 45.5 & 97.4 \\
\hline Apgar 1-min<7 & 1 & 7 & $<0.001 * * *$ & 87.5 & 90.5 & 63.6 & 97.4 \\
\hline Apgar 5-min $<7$ & 0 & 5 & $<0.001 * * *$ & 100 & 86.7 & 45.5 & 100 \\
\hline Fetal distress & 0 & 1 & 0.220 & 100 & 79.6 & 9.1 & 100 \\
\hline Poor outcomes $\ddagger$ & 3 & 10 & $<0.001 * * *$ & 76.9 & 97.3 & 90.9 & 92.3 \\
\hline Group & $\begin{array}{l}\text { Normal UA } \\
\text { Doppler } \\
(\mathrm{n}=44) ; \mathrm{n}\end{array}$ & $\begin{array}{l}\text { Abnormal UA } \\
\text { Doppler }(\mathrm{n}=6) \text {; } \\
\mathrm{n}\end{array}$ & p-value & $\begin{array}{l}\text { Sensitivity } \\
\text { of UA; \% }\end{array}$ & $\begin{array}{l}\text { Specificity } \\
\text { of UA; } \%\end{array}$ & $\begin{array}{l}\text { Positive } \\
\text { Predictive Value; } \\
\%\end{array}$ & $\begin{array}{l}\text { Negative } \\
\text { Predictive Value; } \\
\%\end{array}$ \\
\hline Acidemia & 0 & 0 & - & - & - & & \\
\hline RDS & 3 & 3 & $0.018^{*}$ & 50 & 93.2 & 50.0 & 93.2 \\
\hline Neonatal death & 1 & 0 & 0.880 & 0 & 97.7 & 0 & 87.7 \\
\hline Hypocalcaemia & 1 & 1 & 0.228 & 50 & 89.6 & 16.6 & 97.7 \\
\hline Hypoglycemia & 6 & 3 & 0.063 & 33.3 & 92.7 & 50.0 & 86.4 \\
\hline NICU admission & 3 & 3 & $0.018^{*}$ & 50 & 93.2 & 50.0 & 93.2 \\
\hline Apgar 1-min<7 & 4 & 4 & $0.004 * *$ & 50 & 95.2 & 66.6 & 90.9 \\
\hline Apgar 5-min $<7$ & 4 & 1 & 0.487 & 20 & 88.9 & 16.6 & 90.9 \\
\hline Fetal distress & 1 & 0 & 0.880 & 0 & 87.7 & 0 & 97.7 \\
\hline Poor outcomes $\ddagger$ & 9 & 4 & $0.033^{*}$ & 30.8 & 94.6 & 66.6 & 79.5 \\
\hline
\end{tabular}

$*<0.05, * *<0.01, * * *<0.001 ; \uparrow$ Respiratory distress syndrome, $\$$ the number of infant with poor outcomes

Table 3. Distribution of NST and UA Doppler results according to neonatal outcomes

\begin{tabular}{|l|l|l|l|l|l|}
\hline \multicolumn{2}{|l|}{ Outcomes } & \multicolumn{2}{l|}{ NST } & \multirow{2}{*}{ p-value } \\
\cline { 3 - 5 } \multicolumn{2}{|c|}{ Good } & Reactive & Nonreactive & \\
\hline \multirow{2}{*}{ Poor } & \multirow{2}{*}{ UA Doppler Doppler } & Normal & $34(91.9 \%)$ & $1(2.7 \%)$ & \multirow{2}{*}{0.859} \\
\cline { 3 - 5 } & & Abnormal & $2(5.4 \%)$ & $0(0 \%)$ & \\
\cline { 3 - 5 } & & Abnormal & $2(15.4 \%)$ & $7(53.8 \%)$ & \\
\hline
\end{tabular}

\section{Discussion}

Women with GDM have higher risks of pre-eclampsia, shoulder dystocia, Caesarean, large for gestational age, and prematurity (21). The primary goal of fetal surveillance is to identify fetuses at risk of adverse outcomes, to minimize fetal morbidity and perinatal mortality (22). In modern obstetric medicine, there are non-invasive methods for evaluation of utero-placental function and fetus. Analysis of the results of our research demonstrates that the NST is a better predictor of adverse neonatal outcomes than the UA Doppler test. In the present research, the sensitivity and specificity of the NST in the prediction of neonatal outcomes is acceptable and higher than the sensitivity and specificity of the UA Doppler. Our results were in harmony with previous studies on pregnancies 
complicated by diabetes mellitus, which concluded that Doppler tests were not suitable for diabetic patients without vascular disease. In London's study conducted on 35 insulin-dependent diabetic women, 50\% of fetuses of women with vascular disease had an abnormal UA Doppler in the third-trimester, compared with $12 \%$ in women with uncomplicated diabetes (23). They recommended Doppler methods for diabetes women with vasculopathy, intrauterine growth restriction, and hypertensive disorders $(23,24)$. Our results were not consistent with previous studies which found that UA S/D index was superior to NST in predicting adverse outcomes $(16,17,25)$. In Bracero's study on 207 women with class A insulin-dependent diabetes mellitus, the UA systolic/diastolic index was better than NST and biophysical profile in identifying pregnancies resulted in adverse outcomes (17). In a randomized trial of 1,360 high risk pregnancies, of which $11 \%$ had diabetes mellitus, it was concluded that in the Doppler group, the rate of cesarean delivery for fetal distress was lower than in the NST group, especially in pregnancies with hypertension and suspected intrauterine growth restriction (16). Devine also compared amniotic fluid index (AFI), NST, biophysical profile, and middle cerebral artery (MCA) to UA Doppler ratio to determine the best predictor of adverse outcomes in postdate pregnancies. They found that an MCA to UA ratio $<1.05$ was an accurate method of predicting postdate-related adverse outcome (25). In addition, two studies concluded that UA systolic to diastolic ratio was a valid method to predict adverse outcomes in GDM women. One research implemented on 67 women with GDM showed that women with elevated UA pulsatility indices before delivery experienced increased perinatal complications and cesarean section for fetal distress (26). The second found a significant positive correlation between systolic to diastolic ratios and serum glucose level $(\mathrm{r}=0.52, \mathrm{p}<0.001)$. They also found that an elevated systolic to diastolic ratio was associated with increased number of stillbirths and neonatal morbidity (27). Some studies claimed that a combination of two or more methods could help in identifying at-risk fetuses. Gupta's study showed that fetal Doppler in combination with biophysical profile and cardiotocograph helped in identifying at-risk fetuses for fetal acidosis and encephalopathy (28). We also examined whether combining two tests could improve its validity. We found that combining two tests could improve perinatal outcome by identifying one excess poorer outcome (7.7\%). Result was in agreement with Subramanian's study on 200 cases of high-risk pregnancies which found that when both tests were abnormal, there was a higher percentage of adverse outcomes as compared to that of either Doppler alone being abnormal or NST alone being non-reactive (29). There are also studies which found that combination of an abnormal UA Doppler velocimetry and abnormal glycosylated hemoglobin (30), or uterine artery velocimetry (31) were strongly associated with adverse pregnancy outcome in pregnancies complicated by diabetes.

\section{Conclusions}

We concluded that the validity of the NST was higher than the UA Doppler in the prediction of neonatal outcomes in women with GDM. In addition, using both tests could improve the result slightly (detect one more case). We recommend that in future studies, the use of more than two methods be examined in the prediction of perinatal outcomes of diabetic women. The weak point of the study is the small sample size, which made it necessary for us to use a weak statistical test. We recommend that a systematic review be performed to answer the questions precisely.

\section{Acknowledgments:}

This study was approved by the research chancellery of Tehran University of Medical Sciences and was performed as a requirement of the fellowship degree in perinatology. We thank all the women who participated in the study.

\section{Conflict of Interest:}

There is no conflict of interest to be declared.

\section{Authors' contributions:}

MEY was the main investigator who wrote the first draft and performed sonographies and NSTs and collected the data. SHN and MSH contributed to the study design. FM analyzed the data, revised the second draft and contributed to the interpretation of data and critical revision of the manuscript.

\section{References:}

1) ACOG Practice Bulletin. Clinical Management Guidelines for Obstetrician-Gynecologists. Gestational Diabetes Mellitus. 2013.

2) Metzger B. Proceedings of the Fourth International Work-shop-Conference on Gestational Diabetes Mellitus. Diabetes Care. Fourth International Work-shop-Conference on Gestational Diabetes Mellitus; 1998.

3) Gestational Diabetes Mellitus. Diabetes Care. 2002; 25: s94-6. doi: 10.2337/diacare.25.2007.S94. 
4) Abdalrahman Almarzouki A. Maternal and neonatal outcome of controlled gestational diabetes mellitus versus high risk group without gestational diabetes mellitus: a comparative study. Med Glas (Zenica). 2013; 10: 70-4. PMID: 23348165

5) Barnes-Powell LL. Infants of diabetic mothers: the effects of hyperglycemia on the fetus and neonate. Neonatal Netw. 2007; 26: 283-90. PMID: 17926658. doi: 10.1891/0730-0832.26.5.283.

6) Landon MB, Gabbe SG. Antepartum fetal surveillance in gestational diabetes mellitus. Diabetes. $1985 ; 34$ Suppl 2: 50-4. PMID: 3888742.

7) Yelikar KA, Prabhu A, Thakre GG. Role of Fetal Doppler and Non-Stress Test in Preeclampsia and Intrauterine Growth Restriction. J Obstet Gynaecol India. 2013; 63: 168-72. doi: 10.1007/s13224-0120322-x.

8) Guzman E, Schulman H, Bracero L, Rochelson B, Farmakides G, Coury A. Uterine-umbilical artery Doppler velocimetry in pregnant women with systemic lupus erythematosus. J Ultrasound Med. 1992; 11: 275-81. doi: 10.7863/jum.1992.11.6.275. PMID: 1608089.

9) Mone F, McAuliffe FM, Ong S. The clinical application of Doppler ultrasound in obstetrics. The Obstetrician \& Gynaecologist. 2015; 17: 13-9. doi: 10.1111/tog.12152.

10) O'Neill E, Thorp J. Antepartum Evaluation of the Fetus and Fetal Well Being. Clin Obstet Gynecol. 2012; 55: 722-30. doi: 10.1097/GRF.0b013e318253b318.

11) Miller DA. Nonstress test and contraction stress test. 2016. Available from: http://www.uptodate.com/contents/nonstress-test-and-contraction-stress-test.

12) Zhan Y, Xiang YM, Qi WH, Wang AM, Chen WP, Shao DH. Remote fetal monitoring for gestational diabetes mellitus. Di Yi Jun Yi Da Xue Xue Bao. 2004; 24: 1053-4, 7. PMID: 15447862.

13) Sharma J, Goyal M. Cardiotocography and diabetic pregnancy. J Pak Med Assoc. 2016; 66: S30-3. PMID: 27582148.

14) Alfirevic Z, Stampalija T, Medley N. Fetal and umbilical Doppler ultrasound in normal pregnancy. Cochrane Database Syst Rev. 2015: CD001450. doi: 10.1002/14651858.CD001450.pub4. PMID: 25874722.

15) Maulik D, Yarlagadda P, Youngblood JP, Ciston P. The diagnostic efficacy of the umbilical arterial systolic/diastolic ratio as a screening tool: a prospective blinded study. Am J Obstet Gynecol. 1990; 162: 1518-23. PMID: 2193516.

16) Williams KP, Farquharson DF, Bebbington M, Dansereau J, Galerneau F, Wilson RD, et al. Screening for fetal well-being in a high-risk pregnant population combparing the nonstress test with umbilical artery Doppler velocimetry: a randomized controlled clinical trial. Am J Obstet Gynecol. 2003; 188: 1366-71. PMID: 12748513.

17) Bracero LA, Figueroa R, Byrne DW, Han HJ. Comparison of umbilical Doppler velocimetry, nonstress testing, and biophysical profile in pregnancies complicated by diabetes. J Ultrasound Med. 1996; 15: $301-8$. PMID: 8683665.

18) Shabani Zanjani M, Nasirzadeh R, Fereshtehnejad SM, Yoonesi Asl L, Alemzadeh SA, Askari S. Fetal cerebral hemodynamic in gestational diabetic versus normal pregnancies: a Doppler velocimetry of middle cerebral and umbilical arteries. Acta Neurol Belg. 2014; 114: 15-23. doi: 10.1007/s13760-013-0221-7.

19) Practice Bulletin No. 137. Gestational diabetes mellitus. Obstet Gynecol. 2013; 122: 406-16. doi: 10.1097/01.AOG.0000433006.09219.f1.

20) Cunningham F, Leveno K, Bloom S, Spong C, Dashe J, Hoffman Bea. Whiliams Obstetrics. Williams Obstetrics. 24 ed. New York: McGraw-Hill; 2014.

21) Fadl HE, Ostlund IK, Magnuson AF, Hanson US. Maternal and neonatal outcomes and time trends of gestational diabetes mellitus in Sweden from 1991 to 2003. Diabet Med. 2010; 27: 436-41. doi: 10.1111/j.1464-5491.2010.02978.x.

22) Kautzky-Willer A, Bancher-Todesca D, Pollak A, Repa A, Lechleitner M, Weitgasser R. Gestational diabetes mellitus. Wien Klin Wochenschr. 2012; 124 Suppl 2: 58-65. doi: 10.1007/s00508-012-0265-3.

23) Landon MB, Gabbe SG, Bruner JP, Ludmir J. Doppler umbilical artery velocimetry in pregnancy complicated by insulin-dependent diabetes mellitus. Obstet Gynecol. 1989; 73: 961-5. PMID: 2657528.

24) Graves CR. Antepartum fetal surveillance and timing of delivery in the pregnancy complicated by diabetes mellitus. Clin Obstet Gynecol. 2007; 50: 1007-13. PMID: 17982344. doi: 10.1097/GRF.0b013e31815a63cc.

25) Devine PA, Bracero LA, Lysikiewicz A, Evans R, Womack S, Byrne DW. Middle cerebral to umbilical artery Doppler ratio in post-date pregnancies. Obstet Gynecol. 1994; 84: 856-60. PMID: 7936526. 
26) Fadda GM, Cherchi PL, D'Antona D, Ambrosini G, Marchesoni D, Capobianco G, et al. Umbilical artery pulsatility index in pregnancies complicated by insulin-dependent diabetes mellitus without hypertension. Gynecol Obstet Invest. 2001; 51: 173-7. doi: 10.1159/000052919. PMID: 11306904.

27) Bracero L, Schulman H, Fleischer A, Farmakides G, Rochelson B. Umbilical artery velocimetry in diabetes and pregnancy. Obstet Gynecol. 1986; 68: 654-8. PMID: 3763078.

28) Gupta A, Mehta S, Fazal TS, Sehgal RR, Gogia A. Predictability of Fetal Doppler, Biophysical Profile, and Cardiotocography for Fetal Acidosis at Birth. Journal of Fetal Medicine. 2014; 1: 143-9. doi: 10.1007/s40556-014-0024-9.

29) Subramanian V, Venkat J, Dhanapal M. Which is Superior, Doppler Velocimetry or Non-stress Test or Both in Predicting the Perinatal Outcome of High-Risk Pregnancies. J Obstet Gynaecol India. 2016; 66: 149-56. doi: 10.1007/s13224-015-0829-z. PMID: 27651594.

30) Bracero LA, Haberman S, Byrne DW. Maternal glycemic control and umbilical artery Doppler velocimetry. J Matern Fetal Neonatal Med. 2002; 12: 342-8. PMID: 12607768. doi: 10.1080/jmf.12.5.342.348.

31) Bracero LA, Jovanovic L, Rochelson B, Bauman W, Farmakides G. Significance of umbilical and uterine artery velocimetry in the well-controlled pregnant diabetic. J Reprod Med. 1989; 34: 273-6. PMID: 2715988. 